\title{
BROAD-BAND IMPEDANCE OF LHC SHIELDED BELLOWS
}

\author{
Misha D’yachkov, TRIUMF, 4004 Wesbrook Mall, Vancouver, B.C., Canada V6T 2A3 \\ and \\ Francesco Ruggiero, CERN, Geneva, Switzerland
}

\begin{abstract}
To compensate for thermal expansion the LHC ring has to accommodate about 2500 bellows which, together with beam position monitors, are the main contributors to the LHC broad-band impedance budget. In order to reduce this impedance to an acceptabe value the bellows have to be shielded. In this paper we compare different designs proposed for the bellows and calculate their transverse and longitudinal wake fields and impedances. Owing to the 3D geometry of the bellows, the code MAFIA was used for the wake field calculations. The results presented in this paper indicate that the latest bellows design, in which shielding is provided by sprung fingers which can slide along the beam screen, has impedances smaller than those previously estimated according to a rather conservative scaling of SSC calculations and LEP measurements. Several failure modes, such as missing fingers and imperfect RF contact, have also been studied.
\end{abstract}

\section{INTRODUCTION}

The bellows are the main contributors to the LHC broadband impedance budget [1]. The LHC bellows design is based on the LEP and the SSC bellows [2]. Studies done for SSC bellows $[3,4]$ have shown that in order to reduce their impedances to acceptable values they have to be shielded. The number of particles per bunch in the LHC is even larger than in the SSC $\left(1.05 \times 10^{11}\right.$ compared to $1.5 \times 10^{10}$ in the SSC) and therefore the same applies to LHC bellows.

LHC beam screen has a race-track shape and, in order to provide even pressure on each finger touching the beam screen (for good RF contact), it has been proposed to make a transition from race-track to circular shape. The LHC bellows design has not been finalized yet and therefore the effect of the transition length on the impedance will be studied in this paper.

\section{ANALYTICAL ESTIMATES}

The fact that all transitions and discontinuities in the bellows are very small compared to the beam screen diameter makes it possible to estimate the impedances analytically. It should also be noted that since the r.m.s. bunch length in the LHC $\left(\sigma_{1} \geq 7 \mathrm{~cm}\right)$ is larger than the beam screen radius ( $b \approx 2 \mathrm{~cm}$ ), the beam spectrum lays well below the cutoff frequency of the beam screen $f_{\text {cutoff }}=2.405 c / 2 \pi b=$ $5.74 \mathrm{GHz}$ and therefore we are mostly interested in the low frequency limits of the impedances.

i) Impedance of a step: The small steps (an order of $1 \mathrm{~mm}$ ) are unavoidable in the area where the sliding fingers touch the beam screen; an imaginary part of the longitudinal impedance $Z_{1}$ of a single step can be estimated is given by the following analytic formula, which can be found in Ref. [5]:

$$
Z_{1} / n=-i Z_{0} \frac{\omega_{0}}{c} \frac{h^{2}}{4 \pi^{2} b}[2 \ln (2 \pi b / h)+1] .
$$

The transverse impedance of an axially symmetric discontinuity can be obtained from the longitudinal one using the formula

$$
Z_{\mathrm{t}}=\frac{2 c}{b^{2} \omega_{0}} \frac{Z_{1}}{n}
$$

The resistive part of the impedances (both longitudinal and transverse) of a small step is very small at the typical beam spectrum and can be disregarded. The impedances of a long pill-box, calculated using formulae 1-2 are shown in Table 1 (we assumed that an average beta-function in the $\mathrm{LHC}$ is $\beta_{\mathrm{av}}=89 \mathrm{~m}$ )

Table 1: Impedance contributions from two small steps in the bellows at the contact areas between fingers and beam screen.

\begin{tabular}{l|lll} 
Step size & $1 \mathrm{~mm}$ & $2 \mathrm{~mm}$ & $3 \mathrm{~mm}$ \\
\hline $\mathrm{L}, \mathrm{nH}$ & 0.0340 & 0.118 & 0.243 \\
$Z_{1} / n, \mathrm{~m} \Omega$ & 0.00240 & 0.00835 & 0.0172 \\
$Z_{\mathrm{t}}, \mathrm{k} \Omega / \mathrm{m}$ & 0.051 & 0.177 & 0.364 \\
\hline $2500 \times Z_{1} / n, \mathrm{~m} \Omega$ & 6.00 & 20.9 & 42.9 \\
$2500 \times Z_{\mathrm{t}} \times \beta_{\mathrm{av}}, \mathrm{M} \Omega$ & 0.127 & 0.443 & 0.910
\end{tabular}

ii) The impedance of a gaps between fingers: gaps between separate fingers as well as possible "missing fingers" also have a very small size compared to the beam pipe radius and therefore their effect can be estimated by calculating low frequency impedances of a narrow slot [5]. We found that low-frequency contribution of the gaps is very small compared to a contribution of the "step" which is unavoidable.

\subsection{Power losses in bellows gaps}

As it will be shown in this report, the impedance for different bellows design is well within the LHC impedance budget and the only effect which should be considered is the effect of power losses in the bellows and consequent heating. It is recommended that the total power loss in all bellows should not exceed $1 \mathrm{~kW} /$ ring [2], this number is much higher than the one used in the SSC design $(P<190 \mathrm{~W})$.

The parasitic loss per bellows equals

$$
P=k_{1} e^{2} N^{2} M f_{\text {rev }},
$$


where $k_{\mathrm{l}}$ is the longitudinal loss factor, $\mathrm{N}$ the number of particles per bunch, $\mathbf{M}$ the number of bunches and $f_{\text {rev }}$ is the revolution frequency. The loss factor is negligible in case of good RF contacts between bellows fingers and beam screen, but even a very small gap can contribute significantly to the power losses. The gap can be treated as a coaxial transmission line and thus we can derive the following formula for parasitic losses due to a gap $\Delta$

$$
P=\frac{\Delta}{b} \frac{Z_{0} c}{4 \pi^{3 / 2} \sigma_{\mathrm{z}}} N^{2} e^{2} M f_{\mathrm{rev}}
$$

Plugging typical LHC parameters we obtain the following expression for the power loss per gap:

$$
P(\mathrm{~W}) \simeq 30 \cdot \Delta(\mathrm{mm}) \text {. }
$$

Therefore, in order to have losses below $1 \mathrm{~kW}$ in 2500 bellows and taking into account that there are two sets of fingers, the maximum allowed gap is about $\Delta=0.006 \mathrm{~mm}$.

\subsection{Power losses in other discontinuities}

Due to the fact that the frequency spectrum of the LHC bunch is concentrated in a region well below cutoff frequency of the pipe and the resistive part of the impedance is very small at these frequencies, we estimate that the power losses from all the steps in the bellows will be less than a few watts per ring and can be disregarded. Power losses in the gaps $(<1 \mathrm{~mm})$ between fingers are also found to be small.

The situation when the RF contact is lost for all fingers in a single bellows is equivalent to the "gap" which was considered in the previous subsection. From Eq. (5) we see that, if we assume $\Delta=1 \mathrm{~mm}$, the power losses in a single bellows may be $30 \mathrm{~W}$ ! However, it is unlikely that the RF contact will be lost at all fingers simultaneously. It is more likely that only one or two fingers break at the same time, therefore we need to estimate the power losses due to a few missing fingers. If the power losses due to the "missing" fingers will be higher than the maximum power of the cooling power in the region near the the broken contact, this may cause overheating of neighboring fingers and their subsequent breakup.

We can estimate the power losses in the case of "missing fingers" by representing them as a long slot. Our estimates show that these losses are negligible (which is consistent with the results of numerical simulations shown below).

\section{NUMERICAL RESULTS}

Due to the distinctive 3D geometry of the proposed LHC bellows designs and in order to be able to compare the results for different bellows models, it has been decided to use the MAFIA T3 module (3D time domain) for all calculations. Unfortunately, due to computing limitations, the minimum mesh size we were able to use in our calculations was only $0.5 \mathrm{~mm}$ in the transverse planes and $1 \mathrm{~mm}$ in the longitudinal direction, i.e., memory limitations were

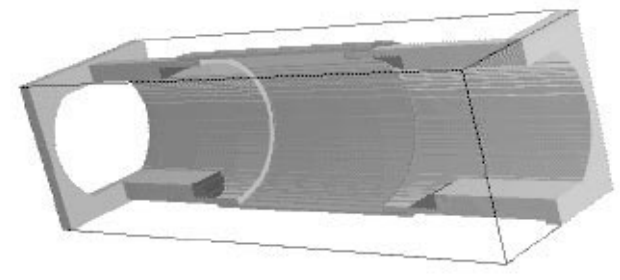

Figure 1: A typical geometry used in the calculations: smooth transition and two steps (pill-box).

our major constraint since the CPU time on the $200 \mathrm{MHz}$ DEC Alpha station was only about 1-2 hours for a typical geometry.

In our simulations we used MAFIA T3 to calculate the wake fields generated by a test Gaussian bunch with charge $\mathrm{Q}=1$ and r.m.s. length $\sigma_{\mathrm{z}}=1 \mathrm{~cm}$, displaced by $5 \mathrm{~mm}$ from the center of the beam pipe in both vertical and horizontal planes and traveling with the speed of light. The wake fields have been calculated with the step $1 \mathrm{~mm}$ and were truncated at $52 \mathrm{~cm}$. The bunch length used in the simulations $\left(\sigma_{1}=1 \mathrm{~cm}\right)$ allowed us to obtain the impedance up to $10-12 \mathrm{GHz}$ (about twice the LHC beam screen cutoff frequency $f_{\text {cutoff }}=5.74 \mathrm{GHz}$ ). On the graphs (Figs. 2-3) the impedance is shown up to $16 \mathrm{GHz}$, together with the spectrum of the $\sigma_{\mathrm{z}}=1 \mathrm{~cm}$ bunch.

The displacement of the beam in the transverse direction was only 10 mesh points, and therefore one should expect additional errors (at least 15-20\%) in the transverse impedances at low frequencies. These errors will be even bigger at higher frequencies. A typical geometry used in the calculations is shown on figure 1.

\subsection{Beam Pipe Transition}

In this section we study the effect of the transition length from the race-track shape to a circle, which is made in order to have the same pressure on each of the sliding fingers.

The low frequency impedances obtained from the results of the simulations for different transition lengths are shown in Table 2. It is interesting to note that the transverse impedances in horizontal and vertical planes are not only different, but have also different signs.

Both transverse and longitudinal impedances decrease sharply if the transition length changes from 0 to $10 \mathrm{~mm}$, and only slightly if it increases from 10 to $35 \mathrm{~mm}$.

Table 2: Low frequency impedances for different transition lengths.

\begin{tabular}{l|cccc} 
Transition & $0 \mathrm{~mm}$ & $10 \mathrm{~mm}$ & $20 \mathrm{~mm}$ & $35 \mathrm{~mm}$ \\
\hline$Z_{l} / n, \mathrm{~m} \Omega$ & 0.0072 & 0.0035 & 0.0034 & 0.0028 \\
$Z_{\mathrm{t}}(v), \mathrm{k} \Omega / \mathrm{m}$ & 0.413 & 0.205 & 0.199 & 0.121 \\
$Z_{\mathrm{t}}(h), \mathrm{k} \Omega / \mathrm{m}$ & -0.276 & -0.138 & -0.129 & -0.079
\end{tabular}



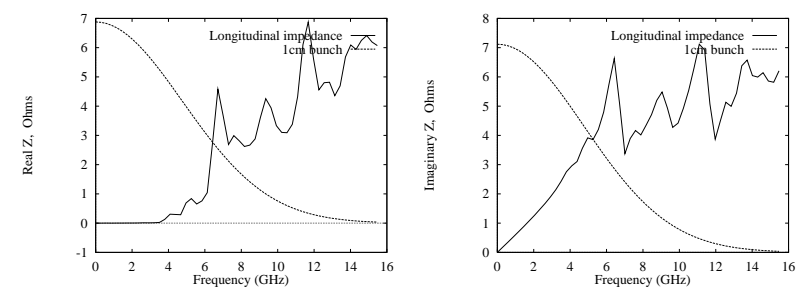

Figure 2: Longitudinal real and imaginary part of impedance for the case of $1 \mathrm{~mm}$ step, $10 \mathrm{~mm}$ transition and 2 missing fingers

\subsection{Transition + step}

The results in the previous section did not include the effect of small steps unavoidable at the point of contact between beam pipe and sliding fingers. In this section we will calculate the impedances of a model bellows which includes smooth transitions and two steps (pill-box).

Using arguments from the previous subsection, the transition length was set to $10 \mathrm{~mm}$ and we calculated the wake fields and the impedances for two different values of the steps, namely $1 \mathrm{~mm}$ and $2 \mathrm{~mm}$ (keeping in mind that though the beam screen is only $1 \mathrm{~mm}$ thick some extra step may be required to allow a good RF contact with the fingers).

The low frequency impedances for the structures considered are presented in Table 3. A "missing finger" was also simulated as a long narrow slot. The width of the slot used in the simulations was $6 \mathrm{~mm}$ (which is in fact equivalent to the case of 2 missing fingers in the same place) though, as one can see from Table 3, the effect of this slot on the overall impedance is very small.

Table 3: Low frequency impedances of the model bellows with smooth transitions of $10 \mathrm{~mm}$ and two steps, for different step sizes and including the case of a missing finger (m.f.).

\begin{tabular}{l|cccc} 
Step size & $0 \mathrm{~mm}$ & $1 \mathrm{~mm}$ & $1 \mathrm{~mm}+$ m.f. & $2 \mathrm{~mm}$ \\
\hline$Z_{1} / n, \mathrm{~m} \Omega$ & 0.0035 & 0.0061 & 0.0062 & 0.0134 \\
$Z_{\mathrm{t}}(v), \mathrm{k} \Omega / \mathrm{m}$ & 0.205 & 0.251 & 0.249 & 0.344 \\
$Z_{\mathrm{t}}(h), \mathrm{k} \Omega / \mathrm{m}$ & -0.138 & -0.088 & -0.094 & -0.007
\end{tabular}

As one can see from Table 3, the longitudinal impedance of the bellows with step $\Delta=2 \mathrm{~mm}$ is only $0.0134 \mathrm{~m} \Omega$, which results in $Z_{1} / n=33.5 \mathrm{~m} \Omega$ for 2500 bellows (i.e., less than half the value of $80 \mathrm{~m} \Omega$ previously allocated for bellows in the LHC impedance budget [1]) and $Z \times \beta_{\mathrm{av}}=$ $0.910 \mathrm{M} \Omega$ also smaller than the value originally used in Ref. [1].

\section{CONCLUSION}

The calculated impedances of the LHC bellows were found to be smaller than the values used in Ref. [1] (based on a rather conservative scaling of SSC calculations and LEP measurements).

It has been found that one of the most significant factors contributing to the impedance of the bellows is the size of

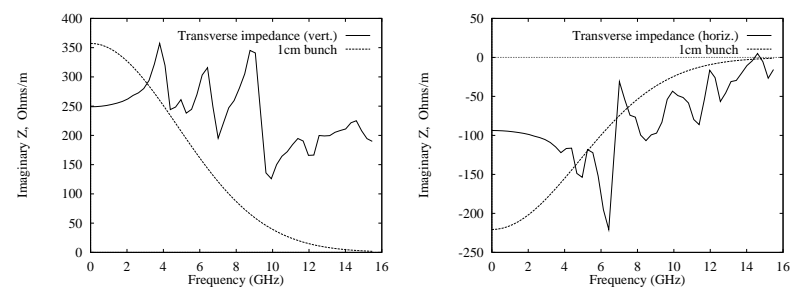

Figure 3: Imaginary part of transverse impedances (vertical and horizontal planes) for the same geometry $(1 \mathrm{~mm}$ step $+10 \mathrm{~mm}$ trans. +2 m.f.).

the step at the point of contact between sliding fingers and beam screen. Therefore additional care should be taken to make this step as small as possible, but retaining at the same time a good RF contact between the fingers and the beam screen, in order to avoid parasitic power losses in the bellows. The gap between the sliding contacts and the beam screen should be less than $0.006 \mathrm{~mm}$ in order to keep the power losses in the bellows $P<1 \mathrm{~kW}$.

The transverse wake fields and impedances of the bellows in the vertical and horizontal planes are not only different, but have also different signs (i.e., focusing in one plane and defocusing in the other). The effect of the gaps between fingers is found to be negligible.

\section{ACKNOWLEDGMENTS}

We would like to thank Alastair Mathewson and Ray Veness, of the LHC Vacuum Group, for useful discussions about the LHC bellows design and for providing us with their latest version.

\section{REFERENCES}

[1] F. Ruggiero, Single-Beam Collective Effects in the LHC, in Proc. "Workshop on Collective Effects in Large Hadron Colliders", Montreux, 1994, eds. E. Keil and F. Ruggiero, Particle Accelerators, Vol. 50, pp. 83-104 (1995).

[2] A. Chao, Summary of the Impedance Working Group, ibid, pp. 1-18 (1995).

[3] K. Ng and J. Bisognano, An Estimate of the Contributions of Bellows to the Impedances and Beam Instabilities of the SSC, ibid, p. 45 (1985).

[4] K.L.F. Bane and R. Ruth, Bellows Wake fields and Transverse Instabilities in the SSC, in "Report of SSC impedance Workshop", SSC-SR-1017, p. 11 (1985).

[5] S. Kurennoy, Coupling Impedance of Pumping Holes, Particle Accelerators, Vol. 39, pp. 1-13 (1992).

[6] S. Kurennoy and G. Stupakov, A New Method for Calculating Low Frequency Coupling Impedance, Particle Accelerators Vol. 45, pp. 95-110 (1994).

[7] S. Kurennoy, Report SSCL-636, Dallas (1993). 